\title{
Addressing Semantic Drift in Question Generation for Semi-Supervised Question Answering
}

\author{
Shiyue Zhang Mohit Bansal \\ UNC Chapel Hill \\ \{shiyue, mbansal\}@es.unc.edu
}

\begin{abstract}
Text-based Question Generation (QG) aims at generating natural and relevant questions that can be answered by a given answer in some context. Existing QG models suffer from a "semantic drift" problem, i.e., the semantics of the model-generated question drifts away from the given context and answer. In this paper, we first propose two semantics-enhanced rewards obtained from downstream question paraphrasing and question answering tasks to regularize the QG model to generate semantically valid questions. Second, since the traditional evaluation metrics (e.g., BLEU) often fall short in evaluating the quality of generated questions, we propose a QA-based evaluation method which measures the QG model's ability to mimic human annotators in generating QA training data. Experiments show that our method achieves the new state-of-theart performance w.r.t. traditional metrics, and also performs best on our QA-based evaluation metrics. Further, we investigate how to use our QG model to augment QA datasets and enable semi-supervised QA. We propose two ways to generate synthetic QA pairs: generate new questions from existing articles or collect QA pairs from new articles. We also propose two empirically effective strategies, a data filter and mixing mini-batch training, to properly use the QG-generated data for QA. Experiments show that our method improves over both BiDAF and BERT QA baselines, even without introducing new articles. ${ }^{1}$
\end{abstract}

\section{Introduction}

In contrast to the rapid progress shown in Question Answering (QA) tasks (Rajpurkar et al., 2016; Joshi et al., 2017; Yang et al., 2018), the task of Question Generation $(\mathrm{QG})$ remains understudied and challenging. However, as an important dual

\footnotetext{
${ }^{1}$ Code and models publicly available at: https:// github.com/Zhangshiyue/QGforQA
}

Context: ...during the age of enlightenment, philosophers such as john locke advocated the principle in their writings, whereas others, such as thomas hobbes, strongly opposed it. montesquieu was one of the foremost supporters of separating the legislature, the executive, and the judiciary...

Gt: who was an advocate of separation of powers? Base: who opposed the principle of enlightenment? Ours: who advocated the principle in the age of enlightenment?

Table 1: An examples of the "semantic drift" issue in Question Generation ("Gt" is short for "ground truth").

task to QA, QG can not only be used to augment QA datasets (Duan et al., 2017), but can also be applied in conversation and education systems (Heilman and Smith, 2010; Lindberg et al., 2013). Furthermore, given that existing QA models often fall short by doing simple word/phrase matching rather than true comprehension (Jia and Liang, 2017), the task of QG, which usually needs complicated semantic reasoning and syntactic variation, should be another way to encourage true machine comprehension (Lewis and Fan, 2019). Recently, we have seen an increasing interest in the QG area, with mainly three categories: Textbased QG (Du et al., 2017; Zhao et al., 2018), Knowledge-Base-based QG (Reddy et al., 2017; Serban et al., 2016), and Image-based QG (Li et al., 2018; Jain et al., 2017). Our work focuses on the Text-based QG branch.

Current QG systems follow an attentionbased sequence-to-sequence structure, taking the paragraph-level context and answer as inputs and outputting the question. However, we observed that these QG models often generate questions that semantically drift away from the given context and answer; we call this the "semantic drift" problem. As shown in Table 1, the baseline QG model generates a question that has almost contrary semantics with the ground-truth question, and the generated phrase "the principle of en- 
lightenment" does not make sense given the context. We conjecture that the reason for this "semantic drift" problem is because the QG model is trained via teacher forcing only, without any high-level semantic regularization. Hence, the learned model behaves more like a question language model with some loose context constraint, while it is unaware of the strong requirements that it should be closely grounded by the context and should be answered by the given answer. Therefore, we propose two semantics-enhanced rewards to address this drift: $\mathbf{Q P P}$ and QAP. Here, QPP refers to Question Paraphrasing Probability, which is the probability of the generated question and the ground-truth question being paraphrases; QAP refers to Question Answering Probability, which is the probability that the generated question can be correctly answered by the given answer. We regularize the generation with these two rewards via reinforcement learning. Experiments show that these two rewards can significantly improve the question generation quality separately or jointly, and achieve the new state-of-the-art performance on the SQuAD QG task.

Next, in terms of QG evaluation, previous works have mostly adopted popular automatic evaluation metrics, like BLEU, METEOR, etc. However, we observe that these metrics often fall short in properly evaluating the quality of generated questions. First, they are not always correlated to human judgment about answerability (Nema and Khapra, 2018). Second, since multiple questions are valid but only one reference exists in the dataset, these traditional metrics fail to appropriately score question paraphrases and novel generation (shown in Table 2). Therefore, we introduce a QA-based evaluation method that directly measures the QG model's ability to mimic human annotators in generating QA training data, because ideally, we hope that the QG model can act like a human to ask questions. We compare different QG systems using this evaluation method, which shows that our semantics-reinforced QG model performs best. However, this improvement is relatively minor compared to our improvement on other QG metrics, which indicates improvement on typical QG metrics does not always lead to better question annotation by QG models for generating QA training set.

Further, we investigate how to use our best QG system to enrich QA datasets and perform semi-supervised QA on SQuADv1.1 (Rajpurkar et al., 2016). Following the back-translation strategy that has been shown to be effective in Machine Translation (Sennrich et al., 2016) and Natural Language Navigation (Fried et al., 2018; Tan et al., 2019), we propose two methods to collect synthetic data. First, since multiple questions can be asked for one answer while there is only one human-labeled ground-truth, we make our QG model generate new questions for existing context-answer pairs in SQuAD training set, so as to enrich it with paraphrased and other novel but valid questions. Second, we use our QG model to label new context-answer pairs from new Wikipedia articles. However, directly mixing synthetic QA pairs with ground-truth data will not lead to improvement. Hence, we introduce two empirically effective strategies: one is a data filter based on QAP (same as the QAP reward) to filter out examples that have low probabilities to be correctly answered; the other is a "mixing mini-batch training" strategy that always regularizes the training signal with the ground-truth data. Experiments show that our method improves both BiDAF (Seo et al., 2016; Clark and Gardner, 2018) and BERT (Devlin et al., 2018) QA baselines by 1.69/1.27 and 1.19/0.56 absolute points on EM/F1, respectively; even without introducing new articles, it can bring 1.51/1.13 and 0.95/0.13 absolute improvement, respectively.

\section{Related Works}

Question Generation Early QG studies focused on using rule-based methods to transform statements to questions (Heilman and Smith, 2010; Lindberg et al., 2013; Labutov et al., 2015). Recent works adopted the attention-based sequenceto-sequence neural model (Bahdanau et al., 2014) for QG tasks, taking answer sentence as input and outputting the question (Du et al., 2017; Zhou et al., 2017), which proved to be better than rulebased methods. Since human-labeled questions are often relevant to a longer context, later works leveraged information from the whole paragraph for QG, either by extracting additional information from the paragraph (Du and Cardie, 2018; Song et al., 2018; Liu et al., 2019) or by directly taking the whole paragraph as input (Zhao et al., 2018; Kim et al., 2018; Sun et al., 2018). A very recent concurrent work applied the large-scale language model pre-training strategy for QG and 
also achieved a new state-of-the-art performance (Dong et al., 2019). However, the above models were trained with teacher forcing only. To address the exposure bias problem, some works applied reinforcement learning taking evaluation metrics (e.g., BLEU) as rewards (Song et al., 2017; Kumar et al., 2018). Yuan et al. (2017) proposed to use a language model's perplexity $\left(R_{P P L}\right)$ and a QA model's accuracy $\left(R_{Q A}\right)$ as two rewards but failed to get significant improvement. Their second reward is similar to our QAP reward except that we use QA probability rather than accuracy as the probability distribution is more smooth. Hosking and Riedel (2019) compared a set of different rewards, including $R_{P P L}$ and $R_{Q A}$, and claimed none of them improved the quality of generated questions. For QG evaluation, even though some previous works conducted human evaluations, most of them still relied on traditional metrics (e.g., BLEU). However, Nema and Khapra (2018) pointed out the existing metrics do not correlate with human judgment about answerability, so they proposed "Q-metrics" that mixed traditional metrics with an "answerability" score. In our work, we will show QG results on traditional metrics, Q-metrics, as well as human evaluation, and also propose a QA-based QG evaluation.

Question Generation for QA As the dual task of QA, QG has been often proposed for improving QA. Some works have directly used QG in QA models' pipeline (Duan et al., 2017; Dong et al., 2017; Lewis and Fan, 2019). Some other works enabled semi-supervised QA with the help of QG. Tang et al. (2017) applied the "dual learning" algorithm (He et al., 2016) to learn QA and QG jointly with unlabeled texts. Yang et al. (2017) and Tang et al. (2018) followed the GAN (Goodfellow et al., 2014) paradigm, taking QG as a generator and QA as a discriminator, to utilize unlabeled data. Sachan and Xing (2018) proposed a self-training cycle between QA and QG. However, these works either reduced the ground-truth data size or simplified the span-prediction QA task to answer sentence selection. Dhingra et al. (2018) collected 3.2M cloze-style QA pairs to pre-train a QA model, then fine-tune with the full groundtruth data which improved a BiDAF-QA baseline. In our paper, we follow the back-translation (Sennrich et al., 2016) strategy to generate new QA pairs by our best QG model to augment SQuAD training set. Further, we introduce a data filter to remove poorly generated examples and a mixing mini-batch training strategy to more effectively use the synthetic data. Similar methods have also been applied in some very recent concurrent works (Dong et al., 2019; Alberti et al., 2019) on SQuADv2.0. The main difference is that we also propose to generate new questions from existing articles without introducing new articles.

\section{Question Generation}

\subsection{Base Model}

We first introduce our base model which mainly adopts the model architecture from the previous state-of-the-art (Zhao et al., 2018). The differences are that we introduce two linguistic features (POS \& NER), apply deep contextualized word vectors, and tie the output projection matrix with the word embedding matrix. Experiments showed that with these additions, our base model results surpass the results reported in Zhao et al. (2018) with significant margins. Our base model architecture is shown in the upper box in Figure 1 and described as follow. If we have a paragraph $p=\left\{x_{i}\right\}_{i=1}^{M}$ and an answer $a$ which is a sub-span of $p$, the target of the QG task is to generate a question $q=\left\{y_{j}\right\}_{j=1}^{N}$ that can be answered by $a$ based on the information in $p$.

Embedding The model first concatenates four word representations: word vector, answer tag embedding, Part-of-Speech (POS) tag embedding, and Name Entity (NER) tag embedding, i.e., $e_{i}=$ $\left[w_{i}, a_{i}, p_{i}, n_{i}\right]$. For word vectors, we use the deep contextualized word vectors from ELMo (Peters et al., 2018) or BERT (Devlin et al., 2018). The answer tag follows the $\mathrm{BIO}^{2}$ tagging scheme.

Encoder The output of the embedding layer is then encoded by a two-layer bi-directional LSTMRNNs, resulting in a list of hidden representations $H$. At any time step $i$, the representation $h_{i}$ is the concatenation of $\overrightarrow{h_{i}}$ and $\overleftarrow{h_{i}}$.

$$
\begin{gathered}
\vec{h}_{i}=\overrightarrow{\operatorname{LSTM}}\left(\left[e_{i} ; \vec{h}_{i-1}\right]\right) \\
\overleftarrow{h}_{i}=\overleftarrow{\operatorname{LSTM}}\left(\left[e_{i} ; \overleftarrow{h}_{i+1}\right]\right) \\
H=\left[\overrightarrow{h_{i}}, \overleftarrow{h_{i}}\right]_{i=1}^{M}
\end{gathered}
$$

\footnotetext{
2"B", for "Begin", tags the start token of the answer span; "I", for "Inside", tags other tokens in the answer span; "O", for "Other", tags other tokens in the paragraph.
} 
Self-attention A gated self-attention mechanism (Wang et al., 2017) is applied to $H$ to aggregate the long-term dependency within the paragraph. $\alpha_{i}$ is an attention vector between $h_{i}$ and each element in $H ; u_{i}$ is the self-attention context vector for $h_{i} ; h_{i}$ is then updated to $f_{i}$ using $u_{i}$; a soft gate $g_{i}$ decides how much the update is applied. $\hat{H}=\left[\hat{h}_{i}\right]_{i=1}^{M}$ is the output of this layer.

$$
\begin{gathered}
u_{i}=H \alpha_{i}, \alpha_{i}=\operatorname{softmax}\left(H^{T} W^{u} h_{i}\right) \\
f_{i}=\tanh \left(W^{f}\left[h_{i} ; u_{i}\right]\right) \\
g_{i}=\operatorname{sigmoid}\left(W^{g}\left[h_{i} ; u_{i}\right]\right) \\
\hat{h}_{i}=g_{i} * f_{i}+\left(1-g_{i}\right) * h_{i}
\end{gathered}
$$

Decoder The decoder is another two-layer unidirectional LSTM-RNN. An attention mechanism dynamically aggregates $\hat{H}$ at each decoding step to a context vector $c_{j}$ which is then used to update the decoder state $s_{j}$.

$$
\begin{gathered}
c_{j}=\hat{H} \alpha_{j}, \alpha_{j}=\operatorname{softmax}\left(\hat{H}^{T} W^{a} s_{j}\right) \\
\tilde{s}_{j}=\tanh \left(W^{c}\left[c_{j} ; s_{j}\right]\right) \\
s_{j+1}=\operatorname{LSTM}\left(\left[y_{j} ; \tilde{s}_{j}\right]\right)
\end{gathered}
$$

The probability of the target word $y_{j}$ is computed by a maxout neural network.

$$
\begin{gathered}
\tilde{o}_{j}=\tanh \left(W^{o}\left[c_{j} ; s_{j}\right]\right) \\
o_{j}=\left[\max \left\{\tilde{o}_{j, 2 k-1}, \tilde{o}_{j, 2 k}\right\}\right]_{k} \\
p\left(y_{j} \mid y_{<j}\right)=\operatorname{softmax}\left(W^{e} o_{j}\right)
\end{gathered}
$$

In practice, we keep the weight matrix $W^{e}$ the same as the word embedding matrix and fix it during training. Furthermore, we apply the same "maxout pointer" proposed by Zhao et al. (2018) to enable the model to copy words from input.

\subsection{Semantics-Reinforced Model}

To address the "semantic drift" problem shown in Table 1, we propose two semantics-enhanced rewards to regularize the generation to focus on generating semantically valid questions.

QPP Reward To deal with the "exposure bias" problem, many previous works directly used the final evaluation metrics (e.g., BLEU) as rewards to train the generation models (Rennie et al., 2017; Paulus et al., 2017). However, these metrics sometimes fail to evaluate equally to question paraphrases and thus provide inaccurate rewards. Hence, we propose to use a pre-trained question paraphrasing classification (QPC) model

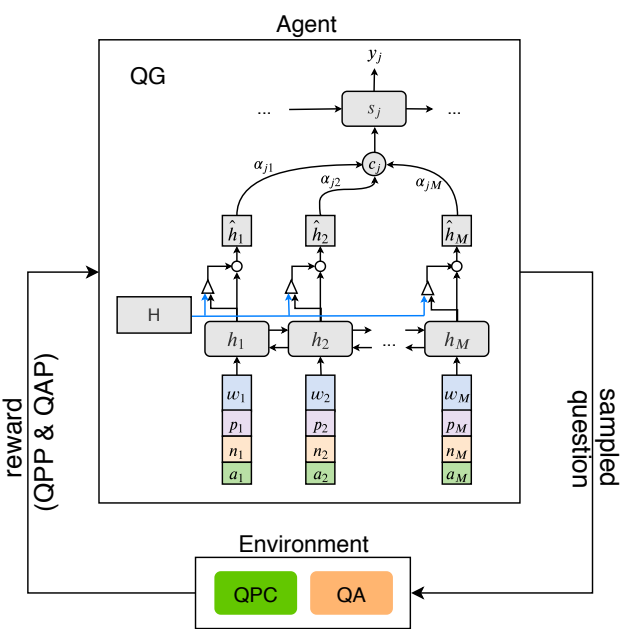

Figure 1: The architecture of our semantics-reinforced QG model.

to provide paraphrasing probability as a reward. Since paraphrasing is more about semantic similarity than superficial word/phrase matching, it treats question paraphrases more fairly (Example 1 in Table 2). Therefore, we first train a QPC model with Quora Question Pairs dataset. Next, we take it as an environment, and the QG model will interact with it during training to get the probability of the generated question and the groundtruth question being paraphrases as the reward.

QAP Reward Two observations motivate us to introduce QAP reward. First, in a paragraph, usually, there are several facts relating to the answer and can be used to ask questions. Neither the teacher forcing or the QPP reward can favor this kind of novel generation (Example 2 in Table 2). Second, we find semantically-drifted questions are usually unanswerable by the given answer. Therefore, inspired by the dual learning algorithm (He et al., 2016), we propose to take the probability that a pre-trained QA model can correctly answer the generated question as a reward, i.e., $p\left(a^{*} \mid q^{s} ; p\right)$, where $a^{*}$ is the ground-truth answer and $q^{s}$ is a sampled question. Using this reward, the model can not only gets positive rewards for novel generation but also be regularized by the answerability requirement. Note that, this reward is supposed to be carefully used because the QG model can cheat by greedily copying words in/near the answer to the generated question. In this case, even though high QAPs are achieved, the model loses the question generation ability.

Policy Gradient To apply these two rewards, we use the REINFORCE algorithm (Williams, 1992) 


\begin{tabular}{|c|c|c|c|c|}
\hline Example 1: Fail to score equally to paraphrases & BLEU4 & Q-BLEU1 & QPP & QAP \\
\hline \multicolumn{5}{|c|}{$\begin{array}{l}\text { Context: ...the university first offered graduate degrees, in the form of a master } \\
\text { of arts ( ma ), in the the } \mathbf{1 8 5 4}-1855 \text { academic year ... } \\
\text { Gt: in what year was a master of arts course first offered ? }\end{array}$} \\
\hline Gen1: in what year did the university first offer a master of arts? & 37.30 & 79.39 & 49.71 & 34.09 \\
\hline Gen2: when did the university begin offering a master of arts? & 29.58 & 47.50 & 46.12 & 18.18 \\
\hline \multicolumn{5}{|l|}{ Example 2: Fail to score appropriately to novel generation } \\
\hline \multicolumn{5}{|c|}{$\begin{array}{l}\text { Context: ...in } \underline{\mathbf{1 9 8 7}} \text {, when some students believed that the observer began to } \\
\text { show a conservative bias, a liberal newspaper, common sense was published... } \\
\text { Gt: in what year did the student paper common sense begin publication? }\end{array}$} \\
\hline Gen1: in what year did common sense begin publication? & 56.29 & 85.77 & 92.28 & 93.94 \\
\hline Gen2: when did the observer begin to show a conservative bias? & 15.03 & 21.11 & 13.44 & 77.15 \\
\hline
\end{tabular}

Table 2: Two examples of where QPP and QAP improve in question quality evaluation.

to learn a generation policy $p_{\theta}$ defined by the $\mathrm{QG}$ model parameters $\theta$. We minimize the loss function $L_{R L}=-E_{q^{s} \sim p_{\theta}}\left[r\left(q^{s}\right)\right]$, where $q^{s}$ is a sampled question from the model's output distribution. Due to the non-differentiable sampling procedure, the gradient is approximated using a single sample with some variance reduction baseline $b$ :

$$
\nabla_{\theta} L_{R L}=-\left(r\left(q^{s}\right)-b\right) \nabla_{\theta} \log p_{\theta}\left(q^{s}\right)
$$

We follow the effective SCST strategy (Rennie et al., 2017) to take the reward of greedy search result $q^{g}$ as the baseline, i.e. $b=r\left(q^{g}\right)$. However, only using this objective to train QG will result in poor readability, so we follow the mixed loss setting (Paulus et al., 2017): $L_{\text {mixed }}=\gamma L_{R L}+$ $(1-\gamma) L_{M L}$. In practice, we find the mixing ratio $\gamma$ for QAP reward should be lower, i.e., it needs more regularization from teacher forcing, so that it can avoid the undesirable cheating issue mentioned above. Furthermore, we also apply the multi-reward optimization strategy (Pasunuru and Bansal, 2018) to train the model with two mixed losses alternately with an alternate rate $n: m$, i.e., train with $L_{\text {mixed }}^{q p p}$ for $n$ mini-batches, then train with $L_{\text {mixed }}^{q a p}$ for $m$ mini-batches, repeat until convergence. $n$ and $m$ are two hyper-parameters.

$$
\begin{aligned}
& L_{\text {mixed }}^{q p p}=\gamma^{q p p} L_{R L}^{q p p}+\left(1-\gamma^{q p p}\right) L_{M L} \\
& L_{\text {mixed }}^{q a p}=\gamma^{q a p} L_{R L}^{q a p}+\left(1-\gamma^{q a p}\right) L_{M L}
\end{aligned}
$$

Experiments show that these two rewards can significantly improve the QG performance separately or jointly, and we achieve new state-of-the-art QG performances, see details in Section 6.

\subsection{QA-Based QG Evaluation}

Inspired by the idea that "a perfect QG model can replace humans to ask questions", we introduce a QA-based evaluation method that measures the quality of a QG model by its ability to mimic human annotators in labeling training data for QA models. The evaluation procedure is described as follows. First, we sample some unlabeled Wikipedia paragraphs with pre-extracted answer spans from HarvestingQA dataset (Du and Cardie, 2018). Second, we make a QG model act as an "annotator" to annotate a question for each answer span. Third, we train a QA model using this synthetic QA dataset. Lastly, we use the QA model's performance on the original SQuAD development set as the evaluation for this QG model. The higher this QA performance is, the better the QG model mimics a human's question-asking ability. We believe that this method provides a new angle to evaluate QG model's quality and also a more reliable way to choose QG models to conduct data augmentation and semi-supervised QA.

\section{Semi-Supervised Question Answering}

Since one of the major goals of developing QG systems is to generate new QA pairs and augment QA datasets, we investigate how to use our QG system to act as a question annotator, collect new QA pairs, and conduct semi-supervised QA. Figure 2 illustrates the overall procedure of our semisupervised QA approach.

\subsection{Synthetic Data Generation}

To generate synthetic QA pairs, we follow the effective "back translation" approach proposed in Neural Machine Translation (NMT) (Sennrich et al., 2016). In NMT, the back translation method first obtains synthetic source sentences by running a pre-trained target-to-source translation model on a monolingual dataset of the target language; then, it combines the synthetic and ground-truth translation pairs to train the desired source-to-target translation model. Similarly, in the QA scenario, 


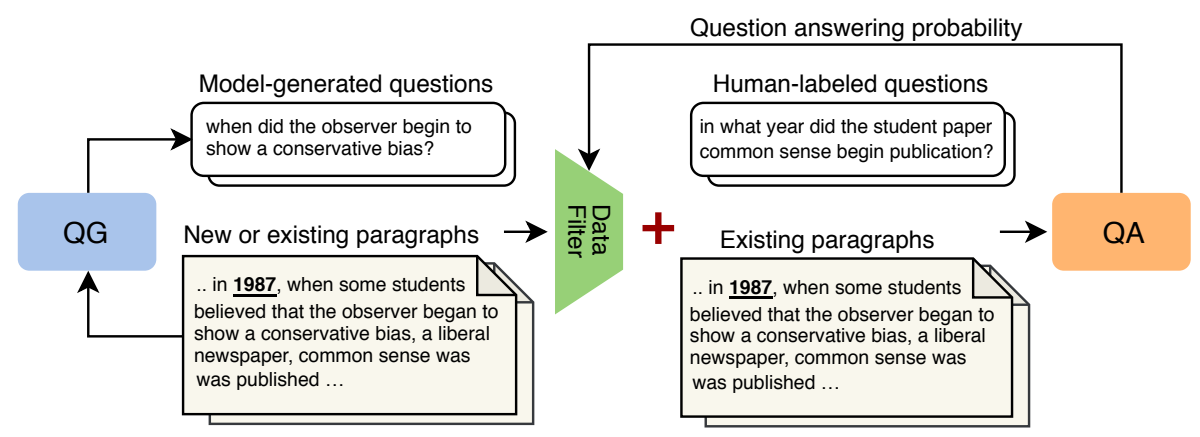

Figure 2: Semi-supervised QA: First, a trained QG model generates questions from new or existing paragraphs building up a synthetic QA dataset; Second, a data filter filters out low-QAP synthetic examples and augment the rest to human-labeled QA pairs; Lastly, the QA model is trained with the enlarged QA dataset.

the paragraph/answer can be viewed as the "target sentence", while the question can be taken as the "source sentence". One tricky difference is that even if the paragraphs can be easily obtained from Wikipedia, there are no answer span labels. Therefore, we use two sources to generate questions from, as discussed below.

Generate from Existing Articles In SQuAD (Rajpurkar et al., 2016), each context-answer pair only has one ground-truth question. However, usually, multiple questions can be asked. The diversity lies in question paraphrasing and different facts in the context that can be used to ask the question. Therefore, without introducing new Wikipedia articles, we make our QG model generate diverse questions for the existing contextanswer pairs in SQuAD training set by keeping the all beam search outputs for each example.

Generate from New Articles To use unlabeled Wikipedia articles for data augmentation, an automatic answer extractor is indispensable. Some previous works have proposed methods to detect key phrases from a paragraph and automatically extract potential answer spans (Yang et al., 2017; Du and Cardie, 2018; Subramanian et al., 2018). Instead of building up our answer extractor, we directly take advantage of the released HarvestingQA dataset. It contains $1.2 \mathrm{M}$ synthetic QA pairs, in which both the answer extractor and the QG model were proposed by Du and Cardie (2018). We use their paragraphs with answer span labels but generate questions with our QG models, and only use their questions for comparison.

\subsection{Synthetic Data Usage}

In practice, we find that directly mixing the synthetic data with the ground-truth data does not improve QA performance. We conjecture the reason is that some poor-quality synthetic examples mislead the learning process of the QA model. Therefore, we propose two empirical strategies to better utilize synthetic data.

QAP Data Filter In "self-training" literature, similar issues have been discussed that using model-labeled examples to train the model will amplify the model's error. Later works proposed co-training or tri-training that uses two or three models as judges and only keeps examples that all models agree on (Blum and Mitchell, 1998; Zhou and Li, 2005). Sachan and Xing (2018) also designed question selection oracles based on curriculum learning strategy in their QA-QG self-training circle. In this paper, we simply design a data filter based on our QAP measure (same definition as the QAP reward) to filter poor-quality examples. We think if one question-answer pair has a low QAP, i.e., the probability of the answer given the question is low, it is likely to be a mismatched pair. Hence, we filter synthetic examples with $Q A P<\epsilon$, where $\epsilon$ is a hyper-parameter that we will tune for different synthetic datasets.

Mixing Mini-Batch Training When conducting semi-supervised learning, we do not want gradients from ground-truth data are overwhelmed by synthetic data. Previous works (Fried et al., 2018; Dhingra et al., 2018) proposed to first pre-train the model with synthetic data and then fine-tune it with ground-truth data. However, we find when the synthetic data size is small (e.g., similar size as the ground-truth data), catastrophic forgetting

\footnotetext{
${ }^{3}$ They actually used the reversed dev-test setup as opposed to the original setup used in Du et al. (2017) and Du and Cardie (2018) (see Section 3.1 in Zhao et al. (2018)). Thus, we also conducted the reversed dev-test setup and our QPP\&QAP model yields BLEU4/METEOR/ROUGE$\mathrm{L}=20.76 / 24.20 / 48.91$.
} 


\begin{tabular}{lllllll}
\hline & BLEU4 & METEOR & ROUGE-L & Q-BLEU1 & QPP & QAP \\
\hline Du and Cardie (2018) & 15.16 & 19.12 & - & - & - & - \\
Zhao et al. (2018) & 16.38 & 20.25 & 44.48 & - & - & - \\
\hline Our baseline (w. ELMo) & 17.00 & 21.44 & 45.89 & 47.80 & 27.29 & 45.15 \\
+ BLEU4 & 17.72 & 22.13 & 46.52 & 49.07 & 27.09 & 45.96 \\
+ METEOR & 17.84 & 22.41 & 46.18 & 49.09 & 26.70 & 46.52 \\
+ ROUGE-L & 17.78 & 22.28 & 46.51 & 49.23 & 27.06 & 46.31 \\
\hline + QPP & 18.25 & 22.62 & 46.45 & 49.59 & $\mathbf{2 8 . 1 3}$ & 47.63 \\
+ QAP & 18.12 & 22.52 & 46.45 & 49.27 & 27.49 & $\mathbf{4 8 . 7 6}$ \\
+ QPP\&QAP & $\mathbf{1 8 . 3 7}$ & $\mathbf{2 2 . 6 5}$ & $\mathbf{4 6 . 6 8}$ & $\mathbf{4 9 . 6 3}$ & 28.03 & 48.37 \\
\hline
\end{tabular}

Table 3: The performance of different QG models.

will happen during fine-tuning, leading to similar results as using ground-truth data only. Thus, we propose a "mixing mini-batch" training strategy, where for each mini-batch we combine half minibatch ground-truth data with half mini-batch synthetic data, which keeps the data mixing ratio to $1: 1$ regardless of what the true data size ratio is. In this way, we can have the training process generalizable to different amounts of synthetic data and keep the gradients to be regularized by groundtruth data.

\section{Experiment Setup}

Datasets For QG, we use the most commonly used SQuAD QG dataset first used by (Du et al., 2017). For QA-based QG evaluation, we obtain unlabeled paragraph and answer labels from HarvestingQA (Du and Cardie, 2018), and have different QG systems to label questions. For semisupervised QA, we use SQuADv1.1 (Rajpurkar et al., 2016) as our base QA task, and split the original development set in half as our development and test set respectively. Plus, we make our QG model generate new questions from both SQuAD and HarvestingQA. We will sample 10\%-100\% examples from HarvestingQA which are denoted by H1-10 in our experiments.

Evaluation Metrics For QG, we first adopt 3 traditional metrics (BLEU4/METEOR/ROUGEL). Second, we apply the new Q-BLEU1 metric proposed by (Nema and Khapra, 2018). Moreover, we conduct a pairwise human evaluation between our baseline and QPP\&QAP model on MTurk. We gave the annotators a paragraph with an answer bold in context and two questions generated by two models (randomly shuffled). We asked them to decide which one is better or nondistinguishable. For both QA-based QG evaluation and semi-supervised QA, we follow the standard evaluation method for SQuAD to use Exact

\begin{tabular}{lll}
\hline QPP\&QAP & Our baseline & Tie \\
\hline 160 & 131 & 9 \\
\hline
\end{tabular}

Table 4: Pairwise human evaluation between our baseline and QPP\&QAP multi-reward model.

\begin{tabular}{llll}
\hline Data & Du and Cardie & Our baseline & QPP \& QAP \\
\hline H1 & $53.20 / 65.47$ & $55.06 / 67.83$ & $\mathbf{5 5 . 8 9 / 6 8 . 2 6}$ \\
H2 & $53.40 / 66.28$ & $56.23 / 69.23$ & $\mathbf{5 6 . 6 9 / 6 9 . 1 9}$ \\
H3 & $53.12 / 65.57$ & $\mathbf{5 7 . 1 4} / 69.39$ & $57.05 / 70.17$ \\
\hline S+H1 & $71.16 / 80.75$ & $71.94 / 81.26$ & $\mathbf{7 2 . 2 0 / 8 1 . 4 4}$ \\
S+H2 & $72.02 / 81.00$ & $72.03 / 81.38$ & $\mathbf{7 2 . 2 2 / 8 1 . 8 1}$ \\
S+H3 & $71.48 / 81.02$ & $72.61 / 81.46$ & $\mathbf{7 2 . 6 9 / 8 2 . 2 2}$ \\
\hline
\end{tabular}

Table 5: The QA-based evaluation results for different QG systems. The two numbers of each item in this table are the EM/F1 scores. All results are the performance on our QA test set. "S" is short for "SQuAD".

Match (EM) and F1.

More details about datasets, evaluation metrics, human evaluation setup, and model implement details are provided in the Appendix.

\section{Results}

\subsection{Question Generation}

Baselines First, as shown in Table 3, our baseline QG model obtains a non-trivial improvement over previous best QG system (Zhao et al., 2018) which proves the effectiveness of our newly introduced setups: introduce POS/NER features, use deep contexturalized word vectors (from ELMo or BERT), and tie output projection matrix with non-trainable word embedding matrix. Second, we apply three evaluation metrics as rewards to deal with the exposure bias issue and improve performance. All the metrics are significantly ${ }^{4}$ $(p<0.001)$ improved except QPP, which supports that high traditional evaluation metrics do not always correlate to high semantic similarity.

\footnotetext{
${ }^{4}$ The significance tests in this paper are conducted following the bootstrap test setup (Efron and Tibshirani, 1994).
} 


\begin{tabular}{|c|c|c|c|c|}
\hline & Filter & Data Size $^{5}$ & EM & F1 \\
\hline \multirow{5}{*}{ 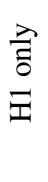 } & $\epsilon=0.0$ & $120 \mathrm{k}$ & 54.55 & 67.91 \\
\hline & $\epsilon=0.2$ & $84 \mathrm{k}$ & 61.18 & 71.65 \\
\hline & $\epsilon=0.4$ & $69 \mathrm{k}$ & 61.97 & 72.48 \\
\hline & $\epsilon=0.6$ & $55 \mathrm{k}$ & 60.38 & 70.51 \\
\hline & $\epsilon=0.8$ & $40 \mathrm{k}$ & 57.47 & 66.48 \\
\hline \multirow{5}{*}{ 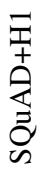 } & $\epsilon=0.0$ & $207 \mathrm{k}$ & 72.97 & 82.18 \\
\hline & $\epsilon=0.2$ & $171 \mathrm{k}$ & 73.88 & 82.72 \\
\hline & $\epsilon=0.4$ & $156 \mathrm{k}$ & 73.47 & 82.62 \\
\hline & $\epsilon=0.6$ & $142 \mathrm{k}$ & 73.96 & 82.81 \\
\hline & $\epsilon=0.8$ & $127 \mathrm{k}$ & 73.65 & 82.77 \\
\hline
\end{tabular}

Table 6: The effect of QAP-based synthetic data filter. We filter out the synthetic data with $Q A P<\epsilon$. All results are the performance on our QA development set.

Semantics-Reinforced Models As shown in Table 3, when using QAP and QQP separately, all metrics are significantly $(p<0.001)$ improved over our baseline and all metrics except ROUGE-L are significantly $(p<0.05)$ improved over the models using traditional metrics as rewards. After applying multi-reward optimization, our model performs consistently best on BLEU4/METEOR/ROUGE-L and Q-BLEU1. Notably, using one of these two rewards will also improve the other one at the same time, but using both of them achieves a good balance between these two rewards without exploiting either of them and results in the consistently best performance on other metrics, which is a new state-ofthe-art result. Human Evaluation Results: Table 4 shows the MTurk anonymous human evaluation study, where we do a pairwise comparison between our baseline and QPP\&QAP model. We collected 300 responses in total, 160 of which voted the QPP\&QAP model's generation is better, 131 of which favors the baseline model, and 9 of which selected non-distinguishable.

QA-Based Evaluation As shown in Table 5, we compare three QG systems using QA-based evaluation on three different amounts of synthetic data and their corresponding semi-supervised QA setups (without filter). It can be observed that both our baseline and our best QG model can significantly improve the synthetic data's QA performance, which means they can act as better "annotators" than the QG model proposed by Du and Cardie (2018). However, our best QG model only has a minor improvement over our baseline model, which means significant improvement over QG metrics does not guarantee significant better question annotation ability.

\begin{tabular}{|c|c|c|c|c|}
\hline & Data & Data Size & EM & $\mathrm{F} 1$ \\
\hline \multirow{5}{*}{$\begin{array}{l}\vec{D} \\
\dot{\infty} \\
\vec{d} \\
\stackrel{0}{ }\end{array}$} & SQuAD & $87 \mathrm{k}$ & 72.52 & 81.79 \\
\hline & + Beam5 & $399 k$ & 74.33 & 83.19 \\
\hline & + Beam10 & $706 \mathrm{k}$ & 74.44 & 83.23 \\
\hline & + Beam15 & $853 \mathrm{k}$ & 74.25 & 82.75 \\
\hline & + DivBeam10 & $595 \mathrm{k}$ & 74.44 & 83.30 \\
\hline \multirow{6}{*}{$\begin{array}{l}\vec{D} \\
\dot{\infty} \\
\vec{d} \\
\stackrel{0}{ }\end{array}$} & $+\mathrm{H} 1$ & $142 \mathrm{k}$ & 73.96 & 82.81 \\
\hline & $+\mathrm{H} 2$ & $255 \mathrm{k}$ & 74.19 & 82.84 \\
\hline & $+\mathrm{H} 4$ & $424 \mathrm{k}$ & 74.42 & 82.82 \\
\hline & + H6 & $506 \mathrm{k}$ & 74.27 & 82.97 \\
\hline & $+\mathrm{H} 8$ & $705 \mathrm{k}$ & 74.64 & 83.14 \\
\hline & $+\mathrm{H} 10$ & $930 \mathrm{k}$ & 74.27 & 82.97 \\
\hline \multirow{4}{*}{ 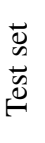 } & SQuAD & $87 \mathrm{k}$ & 71.92 & 81.26 \\
\hline & + Beam 10 & $706 \mathrm{k}$ & 73.43 & 82.39 \\
\hline & $+\mathrm{H} 8$ & $705 \mathrm{k}$ & 73.61 & 82.53 \\
\hline & + Beam10 + H8 & $1.3 \mathrm{M}$ & 73.43 & 82.11 \\
\hline
\end{tabular}

Table 7: The results of our semi-supervised QA method using a BiDAF-QA model.

\begin{tabular}{llll}
\hline Methods & New Data Size & EM & F1 \\
\hline Dhingra et al. base & 0 & 71.54 & 80.69 \\
+Cloze & $3.2 \mathrm{M}$ & 71.86 & 80.80 \\
\hline Our base & 0 & 72.19 & 81.52 \\
+Beam10 & 0 & 73.93 & 82.81 \\
+H8 & $705 \mathrm{k}$ & 74.12 & 82.83 \\
\hline
\end{tabular}

Table 8: The comparison with the previous semisupervised QA method. All results are the performance on the full development set of SQuAD, i.e., our QA test + development set.

\subsection{Semi-Supervised Question Answering}

Effect of the data filter As shown in Table 6, when using synthetic data only, adding the data filter can significantly improve QA performance. In terms of semi-supervised QA, the improvement is relatively smaller, due to the regularization from ground-truth data, but still consistent and stable.

Semi-Supervised QA results Table 7 demonstrates the semi-supervised QA results. Without introducing new articles, we keep beam search outputs as additional questions. It can be seen that using beam search with beam size 10 (+Beam10) improves the BiDAF-QA baseline by $1.51 / 1.13$ absolute points on the testing set. With introducing new articles, the best performance $(+\mathrm{H} 8) \mathrm{im}-$ proves the BiDAF-QA baseline by 1.69/1.27 absolute points on the testing set. We also combine the two best settings (Beam10+H8), but it does not perform better than using them separately.

We conduct two ablation studies on the development set. First, we compare beam search with

\footnotetext{
5"Data Size" counts the total number of examples in training set (after filter). In Table 8, "New Data Size" only counts \# examples generated from articles outside SQuAD.
} 


\begin{tabular}{lllllll|l}
\hline & BLEU4 & METEOR & ROUGE-L & Q-BLEU1 & QPP & QAP & QA-Eval (H1) \\
\hline Du and Cardie (2018) & 15.16 & 19.12 & - & - & - & - & $55.11 / 66.40$ \\
\hline Our baseline (w. BERT) & 18.05 & 22.41 & 46.57 & 49.38 & 29.08 & 54.61 & $58.63 / 69.97$ \\
+ QPP & 18.51 & 22.87 & 46.65 & 49.97 & $\mathbf{3 0 . 1 4}$ & 55.67 & $\mathbf{6 0 . 4 9 / 7 1 . 8 1}$ \\
+ QAP & $\mathbf{1 8 . 6 5}$ & $\mathbf{2 2 . 9 1}$ & $\mathbf{4 6 . 7 6}$ & $\mathbf{5 0 . 0 9}$ & 30.09 & $\mathbf{5 7 . 5 1}$ & $60.12 / 71.14$ \\
+ QPP \& QAP & 18.58 & 22.87 & 46.76 & 50.01 & 30.10 & 56.39 & $59.11 / 70.87$ \\
\hline
\end{tabular}

Table 9: The performance of our stronger BERT-QG models.

\begin{tabular}{|c|c|c|c|c|}
\hline & Data & Data Size & EM & $\mathrm{F} 1$ \\
\hline \multirow{3}{*}{ 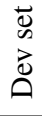 } & SQuAD & $87 \mathrm{k}$ & 81.88 & 88.80 \\
\hline & + Beam 10 & $668 \mathrm{k}$ & 82.34 & 88.97 \\
\hline & + H10 & $664 \mathrm{k}$ & 82.88 & 89.53 \\
\hline \multirow{4}{*}{ 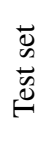 } & SQuAD & $87 \mathrm{k}$ & 80.25 & 88.23 \\
\hline & + Beam10 & $668 \mathrm{k}$ & 81.20 & 88.36 \\
\hline & $+\mathrm{H} 10$ & $664 \mathrm{k}$ & 81.03 & 88.79 \\
\hline & + Beam $10+\mathrm{H} 10$ & $1.2 \mathrm{M}$ & 81.44 & 88.72 \\
\hline
\end{tabular}

Table 10: The results of our semi-supervised QA method using a stronger BERT-QA model.

different beam sizes and diverse beam search $(\mathrm{Li}$ et al., 2016), but all of them perform similarly. Second, increasing the size of synthetic data from $\mathrm{H} 1$ to $\mathrm{H} 10$, the performance saturates around $\mathrm{H} 2-$ $\mathrm{H} 4$. We also observed that when using a big synthetic data, e.g., H10, the model converges even before all examples were used for training. Based on these results, we conjecture that there is an upper bound of the effect of synthetic data which might be limited by the QG quality. To further improve the performance, more diverse and tricky questions need to be generated. To show how QG models help or limit the QA performance, we include some synthetic QA examples in Appendix. Finally, we compare our semi-supervised QA methods with Dhingra et al. (2018). As shown in Table 8, with no or less new data injection, our methods achieve larger improvements over a stronger baseline than their method.

\subsection{QG and QA Results with BERT}

The Bidirectional Encoder Representations from Transformers (BERT) (Devlin et al., 2018) has recently improved a lot of NLP tasks by substantial margins. To verify if our improvements still hold on BERT-based baselines, we propose a BERTQG baseline and test our two semantics-enhanced rewards; further, we conduct our semi-supervised QA method on a BERT-QA baseline.

BERT-QG Without modifying our QG model's architecture, we simply replaced ELMo used above with BERT. Table 9 shows that our BERTQG baseline improves previous ELMo-QG base- line by a large margin; meanwhile, our QPP/QAP rewards significantly improve the stronger $Q G$ baseline and achieve the new state-of-the-art QG performance w.r.t both traditional metrics and QAbased QG evaluation. One difference is that the QAP-only model has the overall best performance instead of the multi-reward model. Note that, we also obtain the QPP and QAP rewards from BERTbased QPC and QA models, respectively.

BERT-QA Using our QAP-reinforced BERTQG model, we apply the same semi-supervised QA method on a BERT-QA baseline. As shown in Table 10, though with smaller margins, our method improves the strong BERT-QA baseline by $1.19 / 0.56$ absolute points on test set; even without introducing new articles, it obtains 0.95/0.13 absolute gains.

\section{Conclusion}

We proposed two semantics-enhanced rewards to regularize a QG model to generate semantically valid questions, and introduced a QA-based evaluation method that directly evaluates a QG model's ability to mimic human annotators in generating QA training data. Experiments showed that our QG model achieves new state-of-the-art performances. Further, we investigated how to use our QG system to augment QA datasets and conduct semi-supervised QA via two synthetic data generation methods along with a data filter and mixing mini-batch training. Experiments showed that our approach improves both BiDAF and BERT QA baselines even without introducing new articles.

\section{Acknowledgments}

We thank the reviewers for their helpful comments and Hao Tan for useful discussions. This work was supported by DARPA (YFA17-D17AP00022), NSF-CAREER Award \#1846185, ONR Grant \#N00014-18-1-2871, and faculty awards from Google, Facebook, and Salesforce. The views contained in this article are those of the authors and not of the funding agency. 


\section{References}

Chris Alberti, Daniel Andor, Emily Pitler, Jacob Devlin, and Michael Collins. 2019. Synthetic qa corpora generation with roundtrip consistency. arXiv preprint arXiv:1906.05416.

Dzmitry Bahdanau, Kyunghyun Cho, and Yoshua Bengio. 2014. Neural machine translation by jointly learning to align and translate. arXiv preprint arXiv:1409.0473.

Avrim Blum and Tom Mitchell. 1998. Combining labeled and unlabeled data with co-training. In Proceedings of the eleventh annual conference on Computational learning theory, pages 92-100. ACM.

Christopher Clark and Matt Gardner. 2018. Simple and effective multi-paragraph reading comprehension. In Proceedings of the 56th Annual Meeting of the Association for Computational Linguistics (Volume 1: Long Papers), pages 845-855.

Alexis Conneau, Douwe Kiela, Holger Schwenk, Loic Barrault, and Antoine Bordes. 2017. Supervised learning of universal sentence representations from natural language inference data. arXiv preprint arXiv:1705.02364.

Michael Denkowski and Alon Lavie. 2014. Meteor universal: Language specific translation evaluation for any target language. In Proceedings of the ninth workshop on statistical machine translation, pages 376-380.

Jacob Devlin, Ming-Wei Chang, Kenton Lee, and Kristina Toutanova. 2018. Bert: Pre-training of deep bidirectional transformers for language understanding. arXiv preprint arXiv: 1810.04805 .

Bhuwan Dhingra, Danish Danish, and Dheeraj Rajagopal. 2018. Simple and effective semi-supervised question answering. In Proceedings of the 2018 Conference of the North American Chapter of the Association for Computational Linguistics: Human Language Technologies, Volume 2 (Short Papers), pages 582-587.

Li Dong, Jonathan Mallinson, Siva Reddy, and Mirella Lapata. 2017. Learning to paraphrase for question answering. In Proceedings of the 2017 Conference on Empirical Methods in Natural Language Processing, pages 875-886.

Li Dong, Nan Yang, Wenhui Wang, Furu Wei, Xiaodong Liu, Yu Wang, Jianfeng Gao, Ming Zhou, and Hsiao-Wuen Hon. 2019. Unified language model pre-training for natural language understanding and generation. arXiv preprint arXiv:1905.03197.

Xinya Du and Claire Cardie. 2018. Harvesting paragraph-level question-answer pairs from wikipedia. In Proceedings of the 56th Annual Meeting of the Association for Computational Linguistics (Volume 1: Long Papers), pages 1907-1917.
Xinya Du, Junru Shao, and Claire Cardie. 2017. Learning to ask: Neural question generation for reading comprehension. In Proceedings of the 55th Annual Meeting of the Association for Computational Linguistics (Volume 1: Long Papers), pages 13421352.

Nan Duan, Duyu Tang, Peng Chen, and Ming Zhou. 2017. Question generation for question answering. In Proceedings of the 2017 Conference on Empirical Methods in Natural Language Processing, pages 866-874, Copenhagen, Denmark. Association for Computational Linguistics.

Bradley Efron and Robert J Tibshirani. 1994. An introduction to the bootstrap. CRC press.

Daniel Fried, Ronghang Hu, Volkan Cirik, Anna Rohrbach, Jacob Andreas, Louis-Philippe Morency, Taylor Berg-Kirkpatrick, Kate Saenko, Dan Klein, and Trevor Darrell. 2018. Speaker-follower models for vision-and-language navigation. In Advances in Neural Information Processing Systems, pages 3314-3325.

Ian Goodfellow, Jean Pouget-Abadie, Mehdi Mirza, Bing Xu, David Warde-Farley, Sherjil Ozair, Aaron Courville, and Yoshua Bengio. 2014. Generative adversarial nets. In Advances in neural information processing systems, pages 2672-2680.

Di He, Yingce Xia, Tao Qin, Liwei Wang, Nenghai Yu, Tie-Yan Liu, and Wei-Ying Ma. 2016. Dual learning for machine translation. In Advances in Neural Information Processing Systems, pages 820-828.

Michael Heilman and Noah A Smith. 2010. Good question! statistical ranking for question generation. In Human Language Technologies: The 2010 Annual Conference of the North American Chapter of the Association for Computational Linguistics, pages 609-617. Association for Computational Linguistics.

Tom Hosking and Sebastian Riedel. 2019. Evaluating rewards for question generation models. arXiv preprint arXiv:1902.11049.

Unnat Jain, Ziyu Zhang, and Alexander Schwing. 2017. Creativity: Generating diverse questions using variational autoencoders. In 2017 IEEE Conference on Computer Vision and Pattern Recognition (CVPR), pages 5415-5424. IEEE.

Robin Jia and Percy Liang. 2017. Adversarial examples for evaluating reading comprehension systems. In Proceedings of the 2017 Conference on Empirical Methods in Natural Language Processing, pages 2021-2031.

Mandar Joshi, Eunsol Choi, Daniel Weld, and Luke Zettlemoyer. 2017. Triviaqa: A large scale distantly supervised challenge dataset for reading comprehension. In Proceedings of the 55th Annual Meeting of the Association for Computational Linguistics (Volume 1: Long Papers), pages 1601-1611. 
Yanghoon Kim, Hwanhee Lee, Joongbo Shin, and Kyomin Jung. 2018. Improving neural question generation using answer separation. arXiv preprint arXiv:1809.02393.

Diederik P Kingma and Jimmy Ba. 2014. Adam: A method for stochastic optimization. arXiv preprint arXiv:1412.6980.

Vishwajeet Kumar, Ganesh Ramakrishnan, and YuanFang Li. 2018. A framework for automatic question generation from text using deep reinforcement learning. arXiv preprint arXiv:1808.04961.

Igor Labutov, Sumit Basu, and Lucy Vanderwende. 2015. Deep questions without deep understanding. In Proceedings of the 53rd Annual Meeting of the Association for Computational Linguistics and the 7th International Joint Conference on Natural Language Processing (Volume 1: Long Papers), volume 1, pages $889-898$.

Mike Lewis and Angela Fan. 2019. Generative question answering: Learning to answer the whole question. In International Conference on Learning Representations (ICLR).

Jiwei Li, Will Monroe, and Dan Jurafsky. 2016. A simple, fast diverse decoding algorithm for neural generation. arXiv preprint arXiv:1611.08562.

Yikang Li, Nan Duan, Bolei Zhou, Xiao Chu, Wanli Ouyang, Xiaogang Wang, and Ming Zhou. 2018. Visual question generation as dual task of visual question answering. In Proceedings of the IEEE Conference on Computer Vision and Pattern Recognition, pages 6116-6124.

Chin-Yew Lin. 2004. Rouge: A package for automatic evaluation of summaries. Text Summarization Branches Out.

David Lindberg, Fred Popowich, John Nesbit, and Phil Winne. 2013. Generating natural language questions to support learning on-line. In Proceedings of the 14th European Workshop on Natural Language Generation, pages 105-114.

Bang Liu, Mingjun Zhao, Di Niu, Kunfeng Lai, Yancheng He, Haojie Wei, and Yu Xu. 2019. Learning to generate questions by learning what not to generate. arXiv preprint arXiv:1902.10418.

Preksha Nema and Mitesh M Khapra. 2018. Towards a better metric for evaluating question generation systems. In Proceedings of the 2018 Conference on Empirical Methods in Natural Language Processing, pages 3950-3959.

Kishore Papineni, Salim Roukos, Todd Ward, and WeiJing Zhu. 2002. Bleu: a method for automatic evaluation of machine translation. In Proceedings of the 40th annual meeting on association for computational linguistics, pages 311-318. Association for Computational Linguistics.
Ramakanth Pasunuru and Mohit Bansal. 2018. Multireward reinforced summarization with saliency and entailment. In Proceedings of the 2018 Conference of the North American Chapter of the Association for Computational Linguistics: Human Language Technologies, Volume 2 (Short Papers), pages 646653.

Romain Paulus, Caiming Xiong, and Richard Socher. 2017. A deep reinforced model for abstractive summarization. arXiv preprint arXiv: 1705.04304.

Matthew Peters, Mark Neumann, Mohit Iyyer, Matt Gardner, Christopher Clark, Kenton Lee, and Luke Zettlemoyer. 2018. Deep contextualized word representations. In Proceedings of the 2018 Conference of the North American Chapter of the Association for Computational Linguistics: Human Language Technologies, Volume 1 (Long Papers), pages 2227-2237.

Pranav Rajpurkar, Jian Zhang, Konstantin Lopyrev, and Percy Liang. 2016. Squad: 100,000+ questions for machine comprehension of text. In Proceedings of the 2016 Conference on Empirical Methods in Natural Language Processing, pages 2383-2392.

Sathish Reddy, Dinesh Raghu, Mitesh M Khapra, and Sachindra Joshi. 2017. Generating natural language question-answer pairs from a knowledge graph using a rnn based question generation model. In Proceedings of the 15th Conference of the European Chapter of the Association for Computational Linguistics: Volume 1, Long Papers, pages 376-385.

Steven J Rennie, Etienne Marcheret, Youssef Mroueh, Jerret Ross, and Vaibhava Goel. 2017. Self-critical sequence training for image captioning. In 2017 IEEE Conference on Computer Vision and Pattern Recognition (CVPR), pages 1179-1195. IEEE.

Mrinmaya Sachan and Eric Xing. 2018. Self-training for jointly learning to ask and answer questions. In Proceedings of the 2018 Conference of the North American Chapter of the Association for Computational Linguistics: Human Language Technologies, Volume 1 (Long Papers), pages 629-640.

Rico Sennrich, Barry Haddow, and Alexandra Birch. 2016. Improving neural machine translation models with monolingual data. In Proceedings of the 54th Annual Meeting of the Association for Computational Linguistics (Volume 1: Long Papers), volume 1, pages 86-96.

Minjoon Seo, Aniruddha Kembhavi, Ali Farhadi, and Hannaneh Hajishirzi. 2016. Bidirectional attention flow for machine comprehension. arXiv preprint arXiv:1611.01603.

Iulian Vlad Serban, Alberto García-Durán, Caglar Gulcehre, Sungjin Ahn, Sarath Chandar, Aaron Courville, and Yoshua Bengio. 2016. Generating factoid questions with recurrent neural networks: 
The $30 \mathrm{~m}$ factoid question-answer corpus. In Proceedings of the 54th Annual Meeting of the Association for Computational Linguistics (Volume 1: Long Papers), volume 1, pages 588-598.

Linfeng Song, Zhiguo Wang, and Wael Hamza. 2017. A unified query-based generative model for question generation and question answering. arXiv preprint arXiv:1709.01058.

Linfeng Song, Zhiguo Wang, Wael Hamza, Yue Zhang, and Daniel Gildea. 2018. Leveraging context information for natural question generation. In Proceedings of the 2018 Conference of the North American Chapter of the Association for Computational Linguistics: Human Language Technologies, Volume 2 (Short Papers), pages 569-574.

Sandeep Subramanian, Tong Wang, Xingdi Yuan, Saizheng Zhang, Adam Trischler, and Yoshua Bengio. 2018. Neural models for key phrase extraction and question generation. In Proceedings of the Workshop on Machine Reading for Question Answering, pages 78-88.

Xingwu Sun, Jing Liu, Yajuan Lyu, Wei He, Yanjun Ma, and Shi Wang. 2018. Answer-focused and position-aware neural question generation. In Proceedings of the 2018 Conference on Empirical Methods in Natural Language Processing, pages 3930 3939.

Hao Tan, Licheng Yu, and Mohit Bansal. 2019. Learning to navigate unseen environments: Back translation with environmental dropout. arXiv preprint arXiv:1904.04195.

Duyu Tang, Nan Duan, Tao Qin, Zhao Yan, and Ming Zhou. 2017. Question answering and question generation as dual tasks. arXiv preprint arXiv:1706.02027.

Duyu Tang, Nan Duan, Zhao Yan, Zhirui Zhang, Yibo Sun, Shujie Liu, Yuanhua Lv, and Ming Zhou. 2018. Learning to collaborate for question answering and asking. In Proceedings of the 2018 Conference of the North American Chapter of the Association for Computational Linguistics: Human Language Technologies, Volume 1 (Long Papers), pages 1564 1574.

Wenhui Wang, Nan Yang, Furu Wei, Baobao Chang, and Ming Zhou. 2017. Gated self-matching networks for reading comprehension and question answering. In Proceedings of the 55th Annual Meeting of the Association for Computational Linguistics (Volume 1: Long Papers), pages 189-198.

Ronald J Williams. 1992. Simple statistical gradientfollowing algorithms for connectionist reinforcement learning. Machine learning, 8(3-4):229-256.

Zhilin Yang, Junjie Hu, Ruslan Salakhutdinov, and William Cohen. 2017. Semi-supervised qa with generative domain-adaptive nets. In Proceedings of the 55th Annual Meeting of the Association for Computational Linguistics (Volume 1: Long Papers), pages 1040-1050.

Zhilin Yang, Peng Qi, Saizheng Zhang, Yoshua Bengio, William W Cohen, Ruslan Salakhutdinov, and Christopher D Manning. 2018. Hotpotqa: A dataset for diverse, explainable multi-hop question answering. arXiv preprint arXiv:1809.09600.

Xingdi Yuan, Tong Wang, Caglar Gulcehre, Alessandro Sordoni, Philip Bachman, Saizheng Zhang, Sandeep Subramanian, and Adam Trischler. 2017. Machine comprehension by text-to-text neural question generation. In Proceedings of the 2nd Workshop on Representation Learning for NLP, pages 15-25.

Yao Zhao, Xiaochuan Ni, Yuanyuan Ding, and Qifa Ke. 2018. Paragraph-level neural question generation with maxout pointer and gated self-attention networks. In Proceedings of the 2018 Conference on Empirical Methods in Natural Language Processing, pages 3901-3910.

Qingyu Zhou, Nan Yang, Furu Wei, Chuanqi Tan, Hangbo Bao, and Ming Zhou. 2017. Neural question generation from text: A preliminary study. In National CCF Conference on Natural Language Processing and Chinese Computing, pages 662-671. Springer.

Zhi-Hua Zhou and Ming Li. 2005. Tri-training: Exploiting unlabeled data using three classifiers. IEEE Transactions on Knowledge and Data Engineering, 17(11):1529-1541.

\section{Appendix}

\section{A Experiment Setup}

\section{A.1 Dataset}

QG For QG, we use the SQuAD-based QG dataset $^{6}$ first introduced by Du et al. (2017) which was the most widely-used QG dataset in previous works (Song et al., 2018; Zhao et al., 2018; Du and Cardie, 2018; Kim et al., 2018; Sun et al., 2018). It was derived from SQuADv1.1 (Rajpurkar et al., 2016). Since the testing set is not open, they sampled $10 \%$ articles from the training set as the testing set, and the original development set is still used for validation.

For the QA-based QG evaluation, we obtain new paragraphs with pre-extracted answer spans from HarvestingQA (Du and Cardie, 2018). Without using their provided questions, we have different QG models act as "annotators" to generate questions, and then use the different QG-labeled synthetic datasets to train QA models. We use the same dev-test setup as described below.

\footnotetext{
${ }^{6}$ https://github.com/xinyadu/nqg/tree/ master/data
} 
QA For QA, we use SQuADv1.1 (Rajpurkar et al., 2016). Previous semi-supervised QA works sampled 10\% from training set as the testing set (Yang et al., 2017; Dhingra et al., 2018). Since we want to use the full training set in semi-supervised QA setup without any data size reduction, we instead split the original development set in half for validation and testing respectively.

For semi-supervised QA, first, without introducing new articles, we generate new questions for SQuAD training set by keeping all beam search outputs. Second, with introducing new articles, we obtain new paragraphs with pre-extracted answer spans from HarvestingQA (Du and Cardie, 2018). Without using their provided questions, we use our best QG model to label questions. Meanwhile, we investigate the influence of synthetic data size, so we sample $10 \%$ to $100 \%$ examples from HarvestingQA, which are denoted as H1-H10 in our experiments.

\section{A.2 Evaluation Metrics}

QG First, we use three traditional automatic evaluation metrics: BLEU4 (Papineni et al., 2002), METEOR (Denkowski and Lavie, 2014), ROUGE-L (Lin, 2004). Second, we adopt the new "Q-metrics" proposed by Nema and Khapra (2018), and we only use "Q-BLEU1" that was shown to have the highest correlation with human judgments on SQuAD. We also take the QPP and QAP rewards as two additional evaluation metrics. Further, we conduct a pairwise human comparison between our baseline and best QG models. Detailed human evaluation setup is described in the next section. For the QA-based QG evaluation, we use the same QA evaluation metrics as follows.

QA Following the standard evaluation method for SQuADv1.1 (Rajpurkar et al., 2016), we use Exact Match (EM) and F1 as two metrics.

\section{A.3 Human Evaluation}

We performed pairwise human evaluation between our baseline and the QPP\&QAP multi-reward model on Amazon Mechanical Turk. We selected human annotators that are located in the US, have an approval rate greater than $98 \%$, and have at least 10,000 approved HITs. We showed the annotators an input paragraph with the answer bold in the paragraph and two questions generated by two QG models (randomly shuffled to anonymize model identities). We then asked them to decide which one is better or choose "nondistinguishable" if they are equally good/bad. We give human three instructions about what is a good question: first, "answerability" - a good question should be answerable by the given answer; "making sense" - a good question should be making sense given the surrounding context; "overall quality" - a good question should be as fluent, non-ambiguous, semantically compact as possible. Ground-truth questions were not provided to avoid simple matching with ground-truth.

\section{B Implementation Details}

QG For ELMo-QG, we first tokenize and obtain the POS/NER tags by Standford Corenlp toolkit ${ }^{7}$, then lower-case the entire dataset. We use 2-layer LSTM-RNNs for both encoder and decoder with hidden size 600. Dropout with a probability of 0.3 is applied to the input of each LSTM-RNN layer. We use the pre-trained character-level word embedding from ELMo (Peters et al., 2018) both as our word embedding and output-projection matrix, and keep it fixed. We use Adam (Kingma and $\mathrm{Ba}, 2014)$ as optimizer with learning rate 0.001 for teacher forcing and 0.00001 for reinforcement learning. Batch size is set to 32 . For stability, we first pre-train the model with teacher forcing until convergence, then fine-tune it with the mixed loss. Hyper-parameters are tuned on development set: $\gamma^{q p p}=0.99, \gamma^{q a p}=0.97$, and $n: m=3: 1$. We use beam search with beam size 10 for decoding and apply a bi-gram/tri-gram repetition penalty as proposed in Paulus et al. (2017).

For BERT-QG, we simply replace the ELMo used above to BERT (Devlin et al., 2018). To match with BERT's tokenization, we use the WordPiece tokenizer to tokenize each word obtained above and extend the POS/NER tags to each word piece. Decoder's word-piece outputs will be mapped to normal words by post-processing. Hyper-parameters are tuned on development set: $\gamma^{q p p}=0.99, \gamma^{q a p}=0.97$, and $n: m=1: 3$.

QA For BiDAF-QA, we implement the BiDAF+Self-attention architecture proposed by Clark and Gardner (2018). We use GRUs for all RNN layers with hidden size 90 for GRUs and 180 for linear layers. Dropout with a probability of 0.2 is applied to the input of each GRU-RNN layer. We optimize the model using Adadelta with

\footnotetext{
${ }^{7}$ https://stanfordnlp.github.io/
} coreNLP / 
Examples generated on SQuAD

Context: ...new york city consists of five boroughs, each of which is a separate county of new york state...

Ground-truth: how many boroughs does new york city contain?

ELMo-QG: how many boroughs make up new york city?

BERT-QG: new york city consists of how many boroughs ?

Context: ...gendn gyatso traveled in exile looking for allies. however, it was not until $\underline{\mathbf{1 5 1 8}}$ that the secular phagmodru ruler captured lhasa from the rinbung, and thereafter the gelug was given rights to conduct the new years prayer...

Ground-truth: when was gelug was given the right to conduct the new years prayer?

ELMo-QG: in what year did the secular phagmodru ruler take over lhasa?

BERT-QG: when did the secular phagmodru ruler capture lhasa from the rinbung ?

Context: ...chopin attended the lower rhenish music festival in aix-la-chapelle with hiller, and it was there that chopin met felix mendelssohn. after the festival, the three visited dsseldorf... they spent what mendelssohn described as "a very agreeable day", playing and discussing music at his piano...

Ground-truth: what two activities did frdric do while visiting for a day in dsseldorf with mendelssohn and hiller?

ELMo-QG: what did mendelssohn do at his piano?

BERT-QG: what did chopin do at his piano?

Context: ...to limit protests, officials pushed parents to sign a document, which forbade them from holding protests, in exchange of money, but some who refused to sign were threatened...

Ground-truth: what has happened to some who refuse to agree to not protest?

ELMo-QG: what did some who refused to sign?

BERT-QG: what did the officials refused to sign ?

Examples generated on HarvestingQA

Context: ...nigeria prior to independence was faced with sectarian tensions and violence... some of the ethnic groups like the ogoni, have experienced severe environmental degradation due to petroleum extraction...

Du and Cardie (2018): what is the main reason for the ethnic groups?

ELMo-QG: why has nigeria experienced severe environmental degradation?

BERT-QG: why have the ogoni experienced severe environmental degradation ?

Context: ...vietnam is located on the eastern indochina peninsula... at its narrowest point in the central qung bnh province, the country is as little as across...

Du and Cardie (2018): where is the country 's country located?

ELMo-QG: in what province is vietnam located ?

BERT-QG: what province is vietnam 's narrowest point ?

Context: ...the ottoman islamic legal system was set up differently from traditional european courts...

Du and Cardie (2018): where was the ottoman islamic legal system set?

ELMo-QG: the ottoman islamic legal system was set up from what ?

BERT-QG: what was the ottoman islamic legal system set up differently from ?

Context: ...the eastern shore of virginia is the site of wallops flight facility, a rocket testing center owned by nasa...

Du and Cardie (2018): what is the eastern shore of virginia owned by?

ELMo-QG: what facility is owned by nasa?

BERT-QG: what is the name of the rocket facility located by nasa?

Table 11: Some synthetic QA examples generated by our QG models.

batch size 64. We also add ELMo to both the input and output of the contextual GRU-RNN layer as proposed in (Peters et al., 2018). To match with QG model's setup, we also apply lower-case on QA datasets.

For BERT-QA, we use the pre-trained uncased BERT-base model $^{8}$ and fine-tune it on QA datasets.

QPC For ELMo-QPC, we follow the model architecture proposed by Conneau et al. (2017). First, two input questions are embedded with ELMo (Peters et al., 2018). Second, the embedded questions are encoded by two 2-layer bidirec-

\footnotetext{
${ }^{8}$ https://github.com/google-research/ bert
}

tional LSTM-RNNs separately with hidden size 512. Next, a max-pooling layer outputs the sentence embedding of each question, denoted by $q_{1}$ and $q_{2}$. Lastly, we input $\left[q_{1}, q_{2},\left|q_{1}-q_{2}\right|, q_{1} * q_{2}\right]$ to an MLP to predict whether these two questions are paraphrases or not. This QPC model is trained using the Quora Question Pairs ${ }^{9}$ dataset. We use Adam (Kingma and Ba, 2014) as optimizer with learning rate 0.0004 and batch size 64 . This model obtained $86 \%$ accuracy on QQP development set.

For BERT-QPC, we also use the pre-trained uncased BERT-base model and fine-tune it on QQP dataset, which obtained 90\% accuracy on QQP development set.

\footnotetext{
${ }^{9}$ https: / $/$ tinyurl.com/y2y8u5ed
} 


\section{Examples}

Table 11 shows some synthetic QA examples generated by our QG models. On SQuAD, the first two examples show our QG models generate some paraphrases or novel questions that enrich the dataset; the last two examples show our QG models generate easier or wrong questions that limit the semi-supervised QA's performance. On HarvestingQA, our QG models can output better questions than Du and Cardie (2018) did but still generate some wrong questions. 